\title{
PFLOTRAN-E4D: A parallel open source PFLOTRAN module for simulating time-lapse electrical resistivity data
}

Timothy C. Johnson ${ }^{1 *}$, Glenn E. Hammond ${ }^{2}$, and Xingyuan Chen ${ }^{1}$ 


\section{Abstract}

Time-lapse electrical resistivity tomography (ERT) is finding increased application for remotely monitoring processes occurring in the near subsurface in three-dimensions (i.e. 4D monitoring). However, there are few codes capable of simulating the evolution of subsurface resistivity and corresponding tomographic measurements arising from a particular process, particularly in parallel and with an open source license. Herein we describe and demonstrate an electrical resistivity tomography module for the PFLOTRAN subsurface flow and reactive transport simulation code, named PFLOTRAN-E4D. The PFLOTRAN-E4D module operates in parallel using a dedicated set of compute cores in a master-slave configuration. At each time step, the master processes receives subsurface states from PFLOTRAN, converts those states to bulk electrical conductivity, and instructs the slave processes to simulate a tomographic data set. The resulting multi-physics simulation capability enables accurate feasibility studies for ERT imaging, the identification of the ERT signatures that are unique to a given process, and facilitates the joint inversion of ERT data with hydrogeological data for subsurface characterization. PFLOTRAN-E4D is demonstrated herein using a field study of stage-driven groundwater/river water interaction ERT monitoring along the Columbia River, Washington, USA. Results demonstrate the complex nature of subsurface electrical conductivity changes, in both the saturated and unsaturated zones, arising from river stage fluctuations and associated river water intrusion into the aquifer. The results also demonstrate the sensitivity of surface based ERT measurements to those changes over time. PFLOTRAN-E4D is available with the PFLOTRAN development version with an open-source license at https://bitbucket.org/pflotran/pflotran-dev . 


\section{Key Words}

hydrogeophysics, time-lapse geophysics, electrical resistivity tomography, groundwater,

25 simulation, multi-physics, parallel, open-source

\section{Introduction}

Electrical Resistivity Tomography (ERT) is a geophysical imaging application whereby the bulk electrical conductivity (i.e. the reciprocal of resistivity) distribution of the subsurface is remotely estimated. ERT has found wide application for understanding the near subsurface because bulk conductivity is governed by both the structural and geochemical properties that characterize subsurface interactions [e.g. Revil et al., 2012]. More recently, time-lapse ERT developments have enabled subsurface processes to be monitored in terms of spatial and temporal changes in bulk conductivity [Binley et al., 2015; Kemna et al., 2006; Singha et al., 2015]. Time-lapse imaging allows the static contributions of bulk conductivity to be removed, thereby revealing the temporal evolution of spatial changes in bulk conductivity. These changes can often be uniquely related to some subsurface process (e.g. fluid transport, geochemical alteration etc.), thereby providing the capability to remotely monitor that process in space and time (see previous references for examples).

Despite the growing popularity of time-lapse ERT imaging, multi-physical simulation codes designed specifically to simulate changes in subsurface bulk conductivity arising from some subsurface process, and then to simulate the corresponding time-lapse ERT data arising from that process, are lacking. Because of this, ERT practitioners have limited tools for investigating the feasibility or performance of a time-lapse ERT imaging campaign for a particular subsurface process prior to field trial. The resulting uncertainty raises the risk of failure and reduces the overall utility of ERT imaging. In addition, ERT survey design, which comprises both electrode layout and measurement sequence, is typically based on standardized applications, as opposed 
47 to being customized to a particular anticipated subsurface process. The capability to accurately

48 simulate the ERT data arising from that process given multiple survey design scenarios would

49 facilitate customized survey designs to improve imaging resolution in both space and time. It

50 would also enable the discovery of critical electrical signatures associated with some processes

51 of interest, and the exploitation of that signature for effective process monitoring. Such a

52 simulator would also facilitate the use of time-lapse ERT data in hydrogeological parameter

53 estimation (deterministic and stochastic) algorithms [Commer et al., 2014; Linde et al., 2006;

54 Rings et al., 2010]. Given the recent advancements in understanding the mechanistic

55 relationships between bulk conductivity and subsurface structural and geochemical states

56 [Revil, 2013; Slater, 2007 and references therein], the capability to accurately derive bulk

57 conductivity from existing subsurface simulators is within reach.

58 In this work we describe a parallel ERT computing module for PFLOTRAN [Hammond et al.,

59 2014], a massively parallel reactive flow and transport model for simulating surface and

60 subsurface processes. Using Message Passing Interface (MPI) libraries [Gropp et al., 1999], a

61 group of processors is created exclusively to accommodate ERT forward modeling. After each

62 time step, PFLOTRAN passes relevant simulation results to this group of modules, which are

63 then transformed to subsurface bulk conductivity, and used to compute a simulated ERT survey

64 for that time step. We demonstrate the code using a groundwater/surface water interaction

65 simulation based upon actual field data. The results show the complex nature of the changes in

66 bulk conductivity arising from stage-driven changes in water table elevation and

67 groundwater/surface water interaction. The results also demonstrate the sensitivity of the ERT

68 data to these processes, thereby validating the capability to monitor them at the field scale. The

69 code and user documentation are available with the PFLOTRAN distribution and associated

70 open source license (https://bitbucket.org/pflotran/pflotran-dev). 


\section{3. Methods}

\subsection{E4D (background, capabilities, parallel structure)}

E4D is a parallel ERT forward modeling and inversion code available with a Berkeley Software Distribution open source license [Johnson and Wellman, 2015a; Johnson et al., 2010] (see https://e4d.pnnl.gov ). The ERT method uses hardware and software to image the bulk electrical conductivity of the subsurface. Bulk electrical conductivity is influenced by physical, biological, and geochemical interactions, making time-lapse images of changes in bulk conductivity useful for monitoring subsurface processes. Measurements are collected using a four-electrode configuration, whereby current is injected between a source and a sink electrode, and the resulting electrical potential is measured between a positive and negative potential electrode. The ratio of the observed potential to the current injected (or some transform thereof) is called the transfer resistance, and constitutes the primary ERT measurement [Daily and Owen, 1991]. In a typical ERT survey, hundreds to tens of thousands of such measurements are strategically collected using array of static electrodes deployed along the surface and/or within boreholes. Time-lapse data sets consist of many identical ERT surveys collected over time to monitor the changes in transfer resistance associated with some subsurface process of interest. Forward modeling is the process whereby the transfer resistance measurements are simulated, given the subsurface bulk conductivity distribution in space and time.

ERT imaging involves inverting the transfer resistance measurements to estimate the bulk conductivity distribution that gave rise the measurements. In comparison to forward modeling, the inversion process is computationally demanding in terms of both CPU load and memory requirements. Details concerning the parallel inversion may be found in Johnson et al. (2010).

3 The work herein focuses on forward modeling, where the subsurface bulk conductivity 4 distribution is generated by converting subsurface states simulated by PFLOTRAN to bulk 
conductivity using a petrophysical transform. The petrophysical transform is defined herein as

96 the relationship between hydrogeological properties and states, and the corresponding

97 subsurface bulk conductivity.

The direct-current electrical potential arising from a given current source is given by the

Poisson equation

$$
\nabla \cdot \sigma_{b}(\mathbf{r}) \nabla \phi(\mathbf{r})=-\nabla \cdot J(\mathbf{r})
$$

where $\mathbf{r}$ is a position vector, $\sigma_{b}$ is effective bulk electrical conductivity, $\phi$ is electrical potential,

and $J$ is the source current density. Given $\sigma, J$, and appropriate boundary conditions, the objective of the forward model is to solve eq. Error! Reference source not found. for $\phi$, which is measured during an ERT experiment. Because potential is a linear function of current density, equation 1 can be solved by superimposing solutions with different current source terms. A pole solution is a solution to equation 1 for a given point source of current with a current sink at infinite distance from the source. Because there are typically many more current source injections than electrodes for a given ERT survey, it is generally more efficient to model the pole solutions for each electrode, and then subtract the pole solution for the sink electrode from the pole solution for the source electrode to simulate the potential field generated by a particular source sink pair. In this way, the number of solutions to equation 1 required to simulate an ERT survey is equal to the number of electrodes instead of the number of measurements.

We use the forward modeling capabilities of E4D to simulate time-lapse ERT surveys in this paper. E4D solves equation 1 using the finite element method on an unstructured tetrahedral mesh. Details concerning numerical aspects of the forward solution can be found in Rucker et al. (2006) and Johnson et al., (2010). Details concerning the parallelization are found in 118 Johnson et al. (2010), and the forward computations are summarized by the flow diagram in 
Figure 1. Numerous aspects of Figure 1 are discussed in the forthcoming sections, including the PFLOTRAN-E4D coupling, mesh interpolation and petrophysical transformation. Of note

121 here are the master-slave configuration of E4D and the highly parallel nature of the E4D forward

122 computations. In particular, E4D has one master process that provides the communication link 123 to PFLOTRAN and orchestrates all E4D computations. Slave nodes accommodate the primary 124 computational burdens and memory requirements, and communicate only with the master node 125 during pre and post solution phases through MPI broadcast calls, making the forward modeling 126 highly scalable.

\subsection{PFLOTRAN (background, capabilities, parallel structure)}

PFLOTRAN is a massively-parallel multi-physics simulator [Hammond et al., 2014] developed and distributed under an open source GNU Lesser General Public License license. It is written in object oriented FORTRAN 2003/2008 and founded upon the PETSc framework

131 [Balay et al., 2015], which provides the underlying parallel data structures and solvers for 132 scalable high performance computing

Although PFLOTRAN is designed for massively parallel computation, the same single code base can run on a single process without recompilation, though available memory may be a limitation. PFLOTRAN's object oriented design facilitates the incorporation of new process 136 models within the code and/or coupling with code developed externally (e.g. E4D). Lichtner et 137 al. (2015) provides a comprehensive overview of PFLOTRAN capability.

For the demonstration in this paper, PFLOTRAN simulated single phase variably saturated

139 flow and solute transport. Single-phase variably saturated flow is based on the Richards'

140 equation with the form

$$
\frac{\partial}{\partial t}(\varphi s \rho)+\nabla \cdot \rho \boldsymbol{q}=0
$$

eq. 2 
142 with water density $\rho$, porosity $\varphi$, and saturation $s$. The Darcy velocity $\boldsymbol{q}$ is given by

$$
\boldsymbol{q}=-\frac{k k_{\tau}}{\mu} \nabla(p-\rho g \mathbf{z})
$$

145 with water pressure $p$, viscosity $\mu$, acceleration of gravity $g$, intrinsic permeability $k$, relative 146 permeability $k_{r}$. Conservative solute transport in the liquid phase is based on the advection147 dispersion equation

$$
\frac{\partial}{\partial t}(\varphi s C)+\nabla \cdot(\boldsymbol{q}-\varphi s D \nabla) C=0
$$

with solute concentration $C$ and dispersion coefficient $D$. PFLOTRAN employs backward Euler

151 time discretization and the Newton-Raphson method (where necessary) to solve for flow and 152 transport state variables over time.

\subsection{PFLOTRAN-E4D coupling}

155 The PFLOTRAN workflow is factored into a tree structure of hierarchical process models

156 (multiphysics processes coupled to numerical methods). As process models are added to

157 PFLOTRAN, factories are adapted or created to accommodate these new process models

158 classes. During simulation initialization, these factories set up process models and link them

159 within a tree data structure (tree graph theory). Each process model has two pointers: one to a

160 child and another to a peer process model as shown in Figure 2. Peer process models

161 synchronize at specified times while descendants play catch up at their own pace. Therefore, 
for the process model tree shown in Figure 3, the master process model A executes first taking as many time steps as necessary to reach a synchronization point. For each A time step, B takes as many time steps as necessary to catch up to A. Once A (and its child B) have reached the synchronization point, $C$ time steps until it reaches the same point in time. Key state variables and parameters are updated between $A$ and $C$ at the synchronization point and 167 between A and B before and after each of A's time steps.

For the PFLOTRAN-E4D coupling, flow serves as the master process model with solute 169 transport as its child, and E4D is the descendant of solute transport as shown in Figure 4.

170 During the initialization, PFLOTRAN sends E4D the porosity distribution, and E4D reads the 171 static Archie's Law (Archie, 1942) parameter distributions (tortuosity factor, cementation factor, 172 and saturation exponent) from a user supplied text file. After each transport step, fluid 173 conductivity and water saturation is passed to E4D, thereby enabling E4D to determine the bulk 174 conductivity distribution at each time step using Archie's Law. If PFLOTRAN is simulating 175 temperature, the temperature distribution is also passed to E4D at each time step. E4D then 176 adjusts the bulk conductivity using a linear transform specified by a user supplied input file.

177 Note that E4D's execution is non-blocking. Once PFLOTRAN hands off the state variables the 178 flow and transport process models can proceed with their execution before E4D has completed 179 its step.

\subsection{Initialization}

Inter-process communications in both PFLOTRAN and E4D are governed using direct MPI

182 calls. Upon initialization, PFLOTRAN divides the available compute cores between PFLOTRAN 183 and E4D as directed by user input. Once the E4D module is initialized, both PFLOTRAN and 184 E4D proceed to read their respective input files and execute pre-simulation setup. This includes 185 generation of the PFIOTRAN-to-E4D mesh interpolation matrix generated by E4D as described 
in section 4.7. The mesh interpolation matrix is used to accurately transfer PFLOTRAN state

187 simulations from the PFLOTRAN mesh to the E4D mesh.

\subsection{PFLOTRAN->E4D data transfer}

Once setup computations are complete, PFLOTRAN begins the subsurface simulation. Upon completion of each time step, PFLOTRAN passes relevant states (e.g. fluid conductivity, saturation, temperature) to the E4D master node and proceeds to the next time step. The E4D master node then determines whether an E4D simulation is required for the current time step, as specified by user input. If a forward simulation is required, the PFLOTRAN states are converted to bulk conductivity and mapped to the E4D mesh by the E4D master node as described in sections 4.6 and 4.7 (Figure 1B). The master node then broadcasts the bulk conductivity distribution to the E4D slave nodes and instructs them to execute a forward run as described in section 4.8 In terms of timing, E4D follows behind PFLOTRAN, executing a forward simulation for the current time step while PFLOTRAN proceeds to simulate the subsurface states for the next time step.

\subsection{Petrophysical Transform}

On its own, bulk electrical conductivity is generally not considered a property of primary importance. However, bulk conductivity is governed by variables that are often considered primary, and are therefore directly simulated by subsurface simulation codes. The direct relationship between these primary variables and bulk conductivity enables spatial and temporal changes in bulk conductivity to be interpreted in terms of corresponding primary properties and processes. The petrophysical transform provides the mathematical link for transforming relevant states simulated by PFLOTRAN to bulk electrical conductivity. 
210 where $a$ is the turtuosity factor, $\Phi$ is porosity, $m$ is the cementation exponent, $s_{w}$ is fluid

211 saturation, $n$ is the saturation exponent, $\sigma_{w}$ is the fluid conductivity.. With the exception of $a$,

$212 m$ and $n$,PFLOTRAN is capable of simulating the state of each parameter on the right hand

213 side of equation 5. Each of these parameters are passed to E4D by PFLOTRANin order to

214 compute the bulk conductivity distribution. PFLOTRAN is also capable of simulating

215 temperature, which, when simulated, is passed to E4D and used to correct the bulk conductivity

216 estimated using equation 5, using a user-provide linear transform.. Once the relevant

217 parameters and state variables are passed to E4D by PFLOTRAN, the E4D master node uses

218 equation 5 and the temperature correction to determine bulk conductivity on the PFLOTRAN

219 mesh, and then interpolates those results to the E4D mesh using the approach described in the 220 next section.

\subsection{Mesh interpolation matrix}

222 Once simulated states are transferred from PFLOTRAN to E4D at a given time step, those 223 states must be transformed to bulk conductivity and mapped to the E4D mesh before a forward 224 run is executed. In addition to the incongruent alignment of the structured mesh used by

225 PFLOTRAN and the unstructured mesh used by E4D, each mesh will typically differ in scale in 226 different regions of the model domain in order to accurately model the differing physics relevant 227 to subsurface fluid flow and electrical current flow from point sources. In order to address these 228 conditions, we integrate the bulk conductivity of the PFLOTRAN mesh over each E4D mesh 229 element. The bulk conductivity is provided at any point in the PFLOTRAN modeling domain 230 using a tri-linear interpolation of the value at the eight PFLOTRAN cell centers surrounding that 
231 point. Figure 5 illustrates the general approach. Let $m_{i}^{e}$ be the bulk conductivity of element $i$ in

232 the E4D mesh. Then

233

$$
m_{i}^{e}=\frac{1}{V_{i}} \int_{V_{i}} m^{c}(\mathbf{r}) d V \approx \frac{1}{n_{k}} \sum_{k=1}^{n_{k}} m_{i, k}^{c}
$$

234 where $V_{i}$ is the volume of E4D mesh element $i, \mathbf{r}$ is a position vector, $m^{c}$ is the bulk conductivity

235 on the PFLOTRAN mesh, $n_{k}$ is the number of subdivisions by which E4D element $i$ is divided

236 for the integral approximation, and $m_{i, k}^{c}$ is the bulk conductivity within element $i$ and subdivision

$237 k$. The value of $m_{i, k}^{c}$ is determined using a tri-linear interpolation of the values of $m^{c}$ at each of

238 the eight PFLOTRAN cell centers surrounding point $i, k$.. This gives

$$
m_{i, k}^{c}=\sum_{j=1}^{n_{c}} m_{j}^{c} W_{i, j, k}
$$

240 where $n_{c}$ is the number of elements in the PFLOTRAN mesh, $m_{j}^{c}$ is the bulk conductivity of

241 element $j$ in the PFLOTRAN mesh, and $W_{i, j, k}$ is the weight given to $m_{j}^{c}$ in determining the value

242 of sub-element $k$ within mesh element $i$ of the E4D mesh.

243 Combining equations 6 and 7 gives the interpolation equation for an E4D mesh element $i$,

$$
m_{i}^{e}=\frac{1}{n_{k}} \sum_{k=1}^{n_{k}} \sum_{j=1}^{n_{c}} m_{j}^{c} W_{i, j, k}
$$

245 Equation 8 is expressed in matrix form as 
247 where $\mathbf{m}^{c}$ is an $n_{c}$ length vector containing the bulk conductivity values on the PFLOTRAN

248 mesh, $\mathbf{m}^{e}$ is a $n_{e}$ length vector containing the bulk conductivity values on the E4D mesh, $n_{e}$ is

249 the number of elements in the E4D mesh, and $\mathbf{M}$ is the $n_{e} x n_{c}$ mesh interpolation matrix.

250 Although $\mathbf{M}$ is sparse, its computation is somewhat demanding. However, $\mathbf{M}$ only needs to be 251 computed once, and its computation is highly parallelizable. As shown in figure $1 \mathrm{~A}, \mathbf{M}$ is 252 computed in parallel by the E4D slave processes during the setup phase. After each slave 253 computes its assigned rows of $\mathbf{M}$, it returns the results to the master process, which assembles 254 the matrix in a sparse format. As shown in figure 1B, the master process is also responsible for 255 conducting the mesh interpolation multiplication represented by equation 9, and transferring the 256 resulting $\mathbf{m}^{e}$ vector of bulk conductivity values to the slave processes in preparation for a 257 forward run.

\subsection{PFLOTRAN-E4D forward modeling}

This section summarizes the forward modeling operations of the PFLOTRAN-E4D module discussed in previous sections. Upon initialization, PFLOTRAN assigns E4D a user specified number of processors. E4D then assigns one processor to be the master process, and the remaining processes as slave processes. The master process reads the E4D input files, passes relevant information to the slave processes, and instructs the slaves to construct and return the mesh interpolation matrix. The master node then instructs the slaves to build the finite element coupling matrix in preparation to solve equation 1 given the bulk conductivity supplied by PFLOTRAN at each user-specified time step.

At each PFLOTRAN time-step, PFLOTRAN sends E4D the simulated subsurface states necessary to derive bulk conductivity using the petrophysical transform given by equation 5 . If user input specifies that an ERT survey should be simulated for the current time step, these 
271 node. The E4D master process then broadcasts the bulk conductivity distribution for the current

272 time step to the slave processes, and instructs the slave processes to compute the solutions for

273 each electrode assigned. The slave processes then extract the relevant electrode potentials

274 from their respective solutions and send them to the master where they are assembled and

275 output to a text file.

276 4. Groundwater/Surface water interaction monitoring simulation

277 We simulated ERT monitoring of a groundwater/surface-water interaction system along the

278 Columbia River at the Hanford Site 300 Area in south-central WA, USA (Figure 6Aquifer

279 sediments bounding the Columbia River are generally coarse grained and highly permeable

280 [Hammond and Lichtner, 2010; Williams et al., 2008]. Coupled with dynamic stage variations,

281 the resulting system is characterized by active stage driven intrusion and retreat of river water

282 into the adjacent unconfined aquifer system.

283 During baseline, low-stage conditions (October-February), the Columbia River is a gaining

284 stream, and the aquifer pore space is occupied by groundwater. As stage levels rise, two

285 processes induce opposing changes in the bulk electrical conductivity of the aquifer (Johnson et

286 al., 2015b). First, the rising water table causes previously unsaturated sediments to become

287 saturated, resulting in an increase in bulk conductivity near the water table (see equation 5).

288 The second change originates in the contrast between lower conductivity river water, and higher

289 conductivity groundwater. As river stage rises river water is driven into the inland aquifer,

290 displacing higher conductivity groundwater, and decreasing bulk conductivity within the

291 saturated zone.

292 Computational meshes

293 Changes in bulk conductivity caused by stage-driven water table fluctuations and river water 
294 intrusion were monitored over time in three-dimensions using ERT with a surface array of 352 295 electrodes (Johnson et al., 2015b). In this example we use PLOTRAN with the E4D module to 296 simulate river water intrusion and the corresponding time-lapse ERT surveys over the first 30 297 days of the experiment. The PFLOTRAN mesh is shown in Figure 7A. Each mesh element is $2982 \mathrm{~m}$ by $2 \mathrm{~m}$ wide in the horizontal direction, and $0.5 \mathrm{~m}$ in the vertical direction, giving $1.6 \mathrm{million}$ 299 elements in total. The mesh is divided into a background host sediment and a higher 300 permeability paleochannel as shown, meant to mimic the paleochannel structures known to 301 govern fluid flow in the 300 Area. The E4D mesh is shown in Figure 7B. The mesh was created 302 to conform to the surface topography and riverbed bathymetry. The boundaries are placed far 303 from electrodes to reduce the effects of the zero-potential subsurface boundary conditions used 304 to solve equation 1. In total, the E4D mesh contains 905,185 elements.

305 To illustrate the alignment between the two meshes, Figure 7C shows the PFLOTRAN mesh 306 superimposed on a transparency of the E4D mesh. Note that the maximum elevation of the 307 water table is $106.7 \mathrm{~m}$, and the PFLOTRAN mesh extends to $110 \mathrm{~m}$ to enable simulation of 308 stage-driven changes in saturation and pore water conductivity in both the saturated and 309 unsaturated zones. PFLOTRAN elements located above the river channel (figure 7C) are 310 inactive in the simulation, such that the PFLOTRAN mesh conforms to the river bathymetry (not 311 shown).

\section{Material Properties}

313 The hydrogeologic properties of the host material and paleochannel used for the PFLOTRAN

314 simulation were selected to provide realistic estimates of field conditions (Lichtner et al., 2010;

315 Williams et al., 2008), and are shown in table 1 below. The unsaturated hydraulic conductivity is 316 computed using the Van Genuchten water retention function [Vangenuchten, 1980] and the 317 Burdine permeability relationship [Burdine, 1953]. 
Table 1. Hydrogeologic material properties of host material and channel shown in Figure 7.

\begin{tabular}{|c|c|c|c|c|c|}
\hline Material & Porosity & $\begin{array}{c}\text { Permeability } \\
\left(\mathrm{m}^{2}\right)\end{array}$ & \multicolumn{2}{|c|}{ Van Genuchten/Burdine Parameters } \\
\cline { 3 - 6 } & & & $\begin{array}{c}\text { Residual } \\
\text { Saturation }\end{array}$ & $\mathrm{m}$ & \\
\hline Host & 0.30 & $1.05 \mathrm{e}-9$ & 0.13 & 0.748 & $1.43 \mathrm{E}-4$ \\
& & & & & \\
\hline Channel & 0.20 & $4.39 \mathrm{e}-9$ & 0.16 & 0.340 & $7.27 \mathrm{E}-4$ \\
& & & & & \\
\hline
\end{tabular}

\section{Boundary and Initial Conditions}

Both pressure and solute concentration boundary and initial conditions are required for the PFLOTRAN simulation. At the northern, western, and southern boundaries, transient

323 (hydrostatic) head conditions were applied using hourly water table elevation measurements

324 [Chen et al. (2013)]. At the eastern boundary transient head boundary conditions are described

325 using river stage measured by a pressure transducer. A conductance value of $10^{-12} \mathrm{~m}$ was

326 applied to the eastern boundary to mimic the damping effect of low-permeability material on the 327 river bed, (Williams et al., 2008, Hammond et al., 2010). A no-flow boundary condition was 328 specified at the bottom of the domain since the domain is underlain by low permeability Ringold 329 Formation materials.

River water intrusion was tracked by introducing a conservative tracer at the river boundary.

331 The concentration boundary conditions for the tracer were set to be free outflow at the northern, 332 western, and southern boundaries, while a constant concentration was maintained at the river 333 boundary. A zero-flux tracer boundary condition was applied at the lower no flow boundary. The 
334 initial flow condition was a hydrostatic pressure distribution based on the water table.The initial 335 conservative tracer concentration was set to be zero over the entire model domain.

As previously described, zero potential boundary conditions are specified on the subsurface boundaries of the E4D mesh. Zero current flux boundary conditions are specified normal to the

338 surface boundary. The E4D elements outside of the PFLOTRAN domain assume a user 339 specified bulk conductivity provided at each time, which is in this case the bulk conductivity 340 provided by the equation 5 at time zero.

\section{Petrophysical Transformation}

342 We assume for the simulation that each variable in equation 5 is known and constant through 343 time, except for fluid conductivity and saturation. . PFLOTRAN simulates saturation and river

344 water concentration in terms of fluid conductivity, and sends each to E4D at each time step.

345 The end-member fluid conductivities are $0.040 \mathrm{~S} / \mathrm{m}$ for river water, and $0.015 \mathrm{~S} / \mathrm{m}$ for

346 groundwater, which were determined by direct measurement.Upon receipt from PFLOTRAN,

347 E4D constructs the bulk conductivity field according to equation 5, maps the filed to the E4D

348 mesh, and executes the forward run. For the simulation, we assumed $a=1, m=1.3$, and $n=2.0$

349 for the Archies parameters.

350 All files required to execute the simulation and reproduce the results given in the next section 351 may be downloaded at (pnnl_sfa_website_address to be placed here upon acceptance).

\section{5. Results}

353 The simulation was executed with 128 processors allocated to PFLOTRAN and 353

354 processors allocated to the E4D module (1 master and 352 slaves, one for each electrode).

355 With this allocation, E4D was able to complete a forward simulation (from mesh interpolation 
through output) within the time required for a single PFLOTRAN time-step, so there was no additional computation time required beyond that required for PFLOTRAN alone. The entire simulation required approximately 3 hours. Given the high permeability of sediments in the simulation, the groundwater table is at all times near the river stage elevation. With this in mind, we demonstrate the results of the PFLOTRAN simulation, petrophysical transformation, and mesh interpolation at two times ( 5 days and 15 days) and at two elevations, one below the water table $(105 \mathrm{~m})$, and one above the water table $(107 \mathrm{~m})$. At 5 and 15 days the river stage 363 is at approximately $105.8 \mathrm{~m}$ and $106.4 \mathrm{~m}$ respectively (Figure 8). Figure 9 shows the change in 364 saturation and river water fraction from baseline conditions at $105 \mathrm{~m}$ and $107 \mathrm{~m}$ simulated by 365 PFLOTRAN, and on the PFLOTRAN mesh (upper panels). The lower panels show the corresponding changes in bulk conductivity derived from the petrophysical transform,

367 interpolated onto to the E4D mesh.

The performance of the mesh interpolation scheme is evident by comparing the change in river water fraction (PFLOTRAN mesh) to the change in bulk conductivity (E4D mesh) at $105 \mathrm{~m}$ for each time shown. Since the subsurface is fully saturated at $105 \mathrm{~m}$, the relationship between a change in fluid conductivity and a change in bulk conductivity is linear, meaning the spatial distribution of the changes should be identical at this elevation. The $105 \mathrm{~m}$ images in Figure 9 show this to be the case, and demonstrate the performance of the mesh interpolation scheme. The same images show the expected behavior of river water intrusion to flow preferentially into the high permeability paleochannel, and the corresponding decrease in bulk conductivity as river water replaces groundwater in the pore space. The uneven river water/groundwater front within the host material (i.e. outside of the channel) is caused by variability in the river bottom bathymetry incorporated into the PFLOTRAN mesh.

The effects of the differing material properties shown in table 1 are highlighted by the change in saturation plots at the $107 \mathrm{~m}$ elevation. At both 5 and 15 days, saturation at $107 \mathrm{~m}$ 
increases within the host material as the water table rises, but saturation increase within the 382 channel is relatively minor. The river water fraction plots show that pore water moving upward 383 into the unsaturated zone is primarily groundwater at 5 days, suggesting the corresponding 384 change in bulk conductivity at 5 days and $107 \mathrm{~m}$ elevation is caused primarily by an increase in saturation with groundwater. Conversely, the change in conductivity at 15 days and $107 \mathrm{~m}$ elevation bares the imprint of the river water intrusion within the saturated zone. The conductivity increase within the vadose zone above the river water intrusion zone is subdued as lower conductivity river water moves into the unsaturated zone through capillary action. Overall, 389 the simulation demonstrates the complex nature of stage driven changes in bulk conductivity associated with this relatively simple synthetic representation of the 300 Area, including complex patterns of conductivity increase within the vadose zone, underlain by decreases in conductivity within the saturated, river water intrusion zone.

Figure 10 shows the E4D-simulated change in electrical potential on the surface from conditions at time zero, and for three different current injection points. The current electrodes for each of the three measurement configurations (M1, M2, and M3) are arranged in a Wenner Alpha array, whereby the two potential measurement electrodes are placed in between the current electrodes, with equal spacing between any two adjacent electrodes. In each case that spacing is $20 \mathrm{~m}$. The locations of each measurement are shown in Figure 10A. M1 is located near the shoreline directly over the paleochannel. M2 is located outside of paleochannel and near the shoreline. M3 is located within the paleochannel, far from the shoreline. For each measurement, the positive current electrode is to the south, the negative current electrode is to 402 the north. Figure 10B shows the change in potential on the ground surface for each 403 measurement, normalized to 1 ampere of injected current, at 5 and 15 days. At 5 days 404 measurements M2 and M3 show similar patterns of potential difference that are caused 405 primarily by the rising water table, because M2 and M3 do not overlie the river water intrusion 
zone at 5 days (see Figure 9). Contrast this to M1, which does overlie the intrusion zone at 5

407 days, and displays a significantly different potential difference pattern compared to M2 and M3.

408 At 15 days, the potential difference distributions for all measurements have changed

409 significantly compared to 5 days. This demonstrates the sensitivity of the ERT measurements to

410 stage driven hydrodynamics, and verifies the corresponding opportunity to image stage-driven

411 changes in electrical conductivity using time-lapse ERT.

412 To further illustrate this point, Figure 11 shows the time series for the potentials measured at

$413 \mathrm{M} 1, \mathrm{M} 2$, and $\mathrm{M} 3$ superimposed on the river stage elevation shown in figure 8 . Note that the

414 simulated potentials are normalized by the injected current to give the transfer resistance. If the

415 support volume for a given measurement becomes less conductive (more resistive), then the

416 transfer resistance will increase, and vice versa. The transfer resistance time series are

417 informative in this regard. M1 displays and increasing trend in transfer resistance, indicating that

418 the subsurface within the support volume for M1 is becoming more resistive, on average. This is

419 consistent with the location of M1, directly over the channel and near the shoreline, where the

420 measurement support volume is primarily influenced by more resistive river water. Note

421 however the influences of water table variations are not lost on the same time series. When

422 river stage (and thus water table) drop, the M1 time series displays a corresponding increase in

423 transfer resistance correspond to the decrease in saturation associated with the falling water

424 table, and vice versa. The same can be observed for M2 and M3. Measurements M2 and M3

425 are primarily influenced by the river stage-driven increase in water table elevation up to about

426 day 10. The rising water table causes an increase in saturation and corresponding decrease in

427 resistivity, as evidenced by the decreasing transfer resistance over that period. After day 10 the

428 transfer resistance begins to increase in M2 and M2 as more resistive river water begins to

429 enter support volume of each measurement. 


\section{Discussion}

In the introduction we stated that a multi-physics simulator capable of modeling the bulk

432 electrical conductivity evolution arising from a given subsurface processes would, among other

433 things, 1) enable practitioners to investigate the feasibility of monitoring that process using ERT,

434 and 2) enable the discovery of critical electrical signatures associated with the process, and the

435 exploitation of that signature for effective process monitoring. The groundwater/surface water

436 interaction simulation presented in sections 5 and 6 is illustrative in this regard. First, the

437 simulation demonstrated that contrasts in bulk conductivity originating from stage-driven river

438 water intrusion provide ERT measurements with adequate sensitivity for monitoring (Figure 11),

439 thereby demonstrating the feasibility of 4D ERT monitoring of river water intrusion and retreat.

440 Second, the simulation revealed the contrasting influences of water table fluctuations and river

441 water intrusion on the bulk conductivity distribution along with the relative magnitudes of those

442 influences in terms of the corresponding ERT measurements (Figure 11). In terms of electrical

443 signatures, those measurements that are not influenced by river water intrusion are

444 characterized by a decrease in transfer resistance with respect to baseline conditions

445 associated with the rising water table. Only measurements that are influenced by river water

446 intrusion experience an increase in transfer resistance compared to baseline conditions. As

447 shown by Wallin et al. (2013) and Johnson et al. (2015b), the ERT data alone do not provide

448 sufficient information to resolve between these competing influences using standard ERT

449 inversion algorithms. Recognition of this fact enabled the development of customized

450 constraints that allowed only increase in bulk conductivity within the vadose zone, and

451 decreases in conductivity within the saturated zone, in relation to baseline conditions (Figure 9).

452 These constraints provided the imaging algorithm with the information necessary to delineate

453 between the influences of water table fluctuations and river water intrusion, thereby enabling

454 effective imaging of the river water intrusion zone. We expect the coupled PFLOTRAN-E4D 
modeling demonstrated here will provide similar utility in other ERT monitoring applications of

456 complex systems.

\section{Conclusion}

458 It is well recognized that geophysical data, particularly time-lapse geophysical data, have

459 the potential to significantly reduce uncertainty in hydrogeologic parameter estimations [Dafflon

460 et al., 2011; Jardani et al., 2013; Linde et al., 2006; Pollock and Cirpka, 2012]. Progress toward

461 informating hydrogeologic inversions with geophysical data is complicated by the general

462 requirement for an accurate field scale petrophysical transform, and by the computational

463 demands of simulating both the hydrological and geophysical observations. The latter can be

464 addressed in part through high performance computing and suitable codes such as

465 PFLOTRAN-E4D. We also note the many advantages of the open source license provided by

466 PFLOTRAN-E4D, which facilitates transparency and collaboration, and enables users to modify

467 the code as needed for custom applications.

\section{7. Acknowledgements}

469 This research was supported by the U.S. Department of Energy (DOE), Office of Biological 470 and Environmental Research (BER), as part of BER's Subsurface Biogeochemistry Research

471 Program (SBR). This contribution originates from the SBR Scientific Focus Area (SFA) at the 472 Pacific Northwest National Laboratory (PNNL).

\section{8. References}

474 Archie, G. E. (1942), The electrical resistivity log as an aid in determining some reservoir 475 characteristics, T Am I Min Met Eng, 146, 54-61. 
476 Balay, S., J. Brown, K. Buschelman, V. Eijkhout, W. D. Gropp, D. Kaushik, M. G. Knepley, L. C. 477 Mclnnes, B. F. Smith, and H. Zhang (2015), PETSc Users Manual, Tech. Rep. ANL-95/11478 Revision 3.5, Argonne Natl. Lab., Argonne, III.

479 Binley, A., S. S. Hubbard, J. A. Huisman, A. Revil, D. A. Robinson, K. Singha, and L. D. Slater 480 (2015), The emergence of hydrogeophysics for improved understanding of subsurface $481 \quad$ processes over multiple scales, Water Resour Res, 51(6), 3837-3866.

482 Burdine, N. T. (1953), Relative Permeability Calculations from Pore Size Distribution Data, T Am $483 \quad$ I Min Met Eng, 198, 71-78.

484 Chen, X., G. E. Hammond, C. J. Murray, M. L. Rockhold, V. R. Vermeul, and J. M. Zachara 485 (2013), Application of ensemble-based data assimilation techniques for aquifer 486 characterization using tracer data at Hanford 300 Area, Water Resour Res, 49(10), 7064$487 \quad 7076$.

488 Daily, W., and E. Owen (1991), Cross-Borehole Resistivity Tomography, Geophysics, 56(8), 489 1228-1235.

490 Gropp, W., E. Lusk, and R. Thakur (1999), Using MPI-2: Advanced Features of the Message$491 \quad$ Passing Interface, MIT Press.

492 Hammond, G. E., and P. C. Lichtner (2010), Field-scale model for the natural attenuation of 493 uranium at the Hanford 300 Area using high-performance computing, Water Resour Res, $494 \quad 46$.

495 Hammond, G. E., P. C. Lichtner, and R. T. Mills (2014), Evaluating the performance of parallel 496 subsurface simulators: An illustrative example with PFLOTRAN, Water Resour Res, 50(1), $497 \quad 208-228$. 
Johnson, T. C., and D. M. Wellman (2015), Accurate modelling and inversion of electrical resistivity data in the presence of metallic infrastructure with known location and dimension, Geophys. J. Int., 202(2), 1096-1108.

Johnson, T., R. Versteeg, J. Thomle, G. Hammond, X. Chen, and J. Zachara(2015b), Four-

Johnson, T. C., R. J. Versteeg, A. Ward, F. D. Day-Lewis, and A. Revil (2010), Improved dimensional electrical conductivity monitoring of stage-driven river water intrusion:

Accounting for water table effects using a transient mesh boundary and conditional inversion constraints, Water Res.. Res., 51, 6177-6196, doi:10.1002/2014WR016129.

Lichtner, P.C., G.E. Hammond, C. Lu, S. Karra, G. Bisht, B. Andre, R.T. Mills and J. Kumar (2015) PFLOTRAN Users Manual, www.pflotran.org.

Kemna, A., A. Binley, F. Day-Lewis, A. Englert, B. Tezkan, J. Vanderborght, H. Vereecken, and hydrogeophysical characterization and monitoring through parallel modeling and inversion of time-domain resistivity and induced-polarization data, Geophysics, 75(4), Wa27-Wa41.

512 Revil, A. (2013), Effective conductivity and permittivity of unsaturated porous materials in the 513 frequency range $1 \mathrm{mHz}-1 \mathrm{GHz}$, Water Res. Res, 49(1), 306-327.

514 Revil, A., M. Karaoulis, T. Johnson, and A. Kemna (2012), Review: Some low-frequency

515 electrical methods for subsurface characterization and monitoring in hydrogeology, $516 \quad$ Hydrogeol J, 20(4), 617-658.

517 Rucker, C., T. Gunther, and K. Spitzer (2006), Three-dimensional modelling and inversion of dc 518 resistivity data incorporating topography - I. Modelling, Geophys J Int, 166(2), 495-505. 
Singha, K., F. D. Day-Lewis, T. Johnson, and L. D. Slater (2015), Advances in interpretation of subsurface processes with time-lapse electrical imaging, Hydrol Process, 29(6), 1549-1576.

Slater, L. (2007), Near surface electrical characterization of hydraulic conductivity: From petrophysical properties to aquifer geometries - A review, Surv. Geophys, 28(2-3), 169-197.

vanGenuchten, M. T. (1980), A Closed-Form Equation for Predicting the Hydraulic Conductivity of Unsaturated Soils, Soil Sci Soc Am J, 44(5), 892-898.

Williams, M. D., M. L. Rockhold, P. D. Thorne, and Y. Chen (2008), Three-Dimensional Groundwater Models of the 300 Area at the Hanford Site, Washington StateRep., Pacific Northwest National Laboratory, Richland, WA.

Commer, M., M. B. Kowalsky, J. Doetsch, G. A. Newman, and S. Finsterle (2014), MPiTOUGH2: A parallel parameter estimation framework for hydrological and hydrogeophysical applications, Comput Geosci-Uk, 65, 127-135.

Dafflon, B., W. Barrash, M. Cardiff, and T. C. Johnson (2011), Hydrological parameter estimations from a conservative tracer test with variable-density effects at the Boise Hydrogeophysical Research Site, Water Resour Res, 47.

Jardani, A., A. Revil, and J. P. Dupont (2013), Stochastic joint inversion of hydrogeophysical 535 data for salt tracer test monitoring and hydraulic conductivity imaging, Adv Water Resour, $536 \quad 52,62-77$

537 Linde, N., J. S. Chen, M. B. Kowalsky, and S. Hubbard (2006), Hydrogeophysical parameter 538 estimation approaches for field scale characterization, Nato Sci S Ss Iv Ear, 71, 9-44.

539 Pollock, D., and O. A. Cirpka (2012), Fully coupled hydrogeophysical inversion of a laboratory 540 salt tracer experiment monitored by electrical resistivity tomography, Water Resour Res, 48. 
541 Rings, J., J. A. Huisman, and H. Vereecken (2010), Coupled hydrogeophysical parameter

542 estimation using a sequential Bayesian approach, Hydrol Earth Syst Sc, 14(3), 545-556.

543

544 Figures

545 


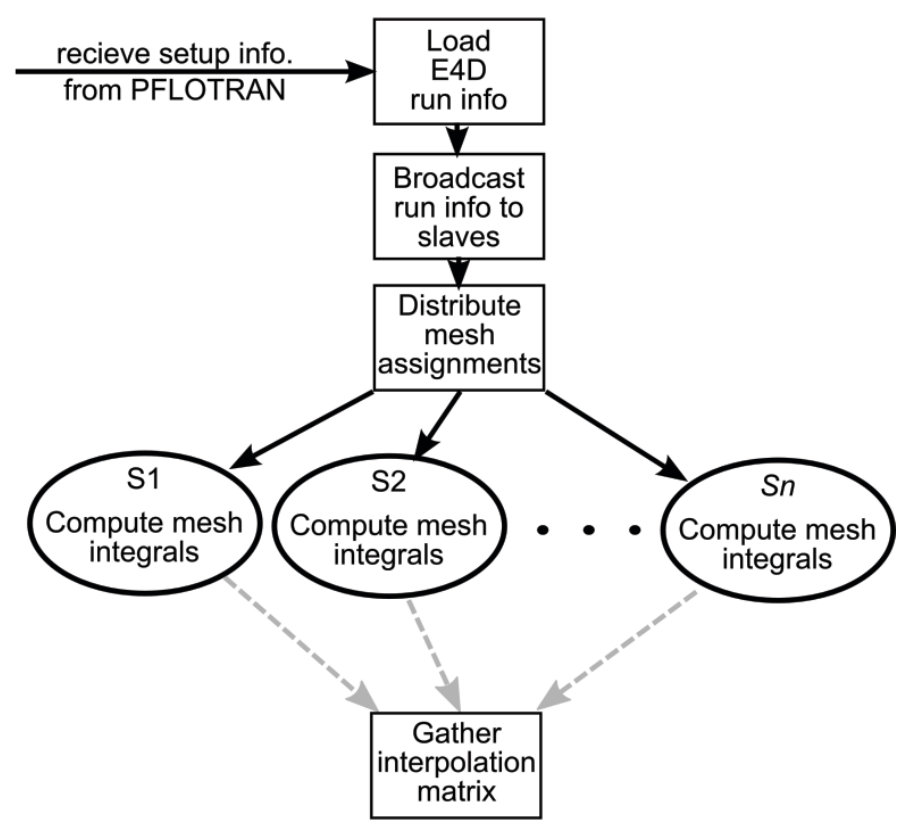

B) Run phase

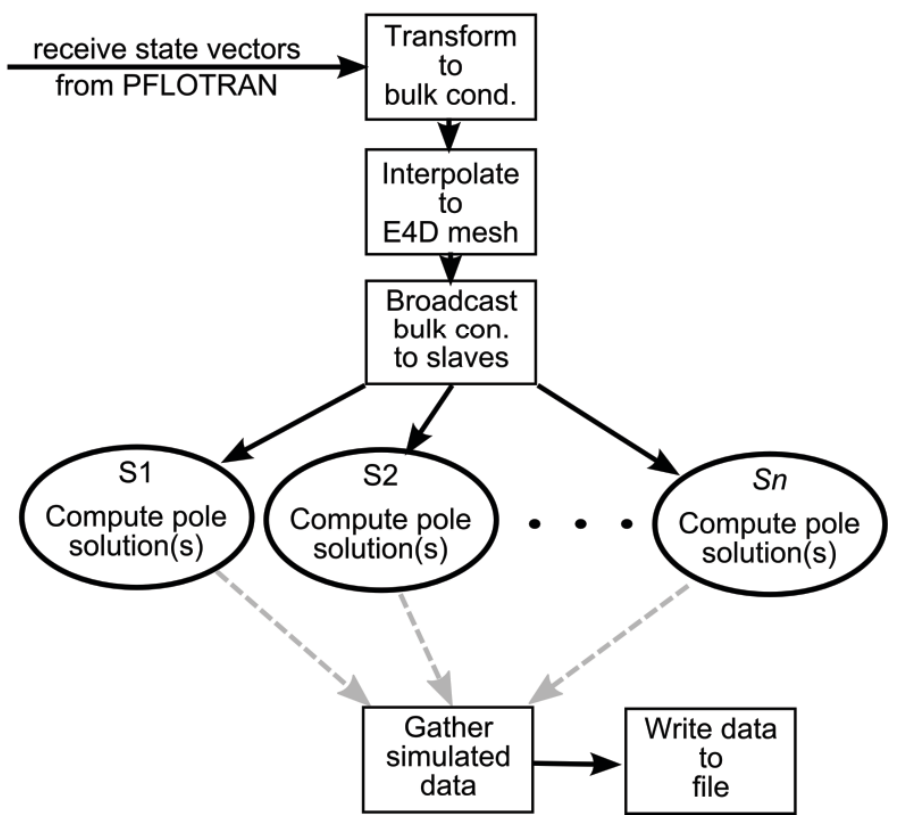

547 Figure 1. Flow diagram showing the setup and run phases of the E4D module. A) In the 548 setup phase, E4D is initialized by PFLOTRAN. The master node receives setup and instructs 
549 the slave nodes to construct the PFLOTRAN-E4D mesh interpolation matrix. B) At each time 550 step, the E4D master node receives subsurface state information from PFLOTRAN, converts

551 that information to bulk conductivity with a user supplied petrophysical transform, maps the bulk

552 conductivity to the E4D mesh, and transfers the results to the slave nodes. The slave nodes

553 compute pole solutions for assigned electrodes, and transfer the relevant potentials to the

554 master node for assembly. The master node assembles the potentials to create the simulated

555 measurements, and writes the results to a file.

556

557 


\section{PFLOTRAN Process Model}

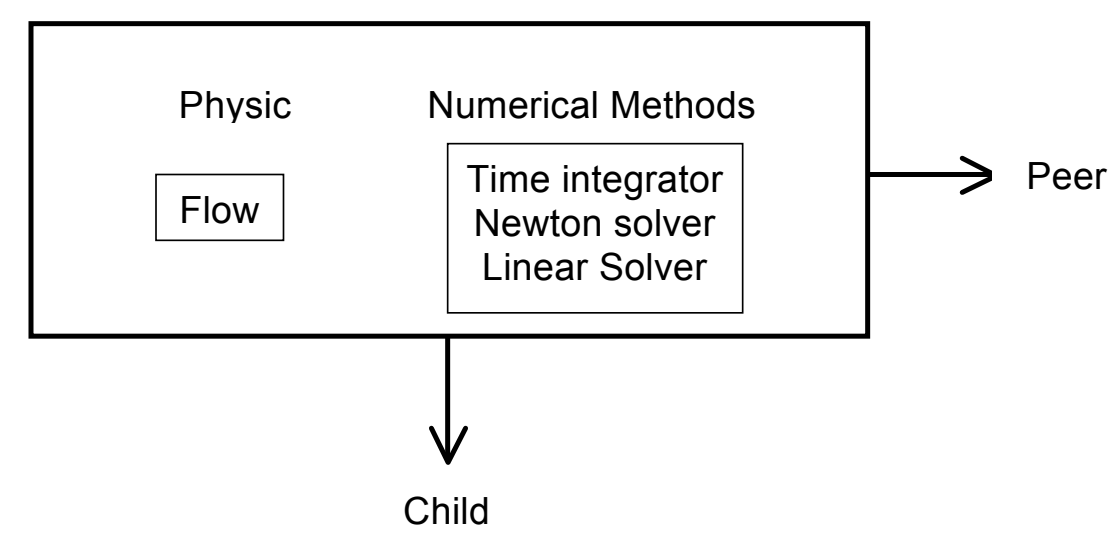

559 Figure 2. The PFLOTRAN process model class coupling physics/chemistry to numerical

560 methods for their solution. Each process model has a pointer to a neighboring peer and child,

561 which enables linkage between process models through a tree data structure (tree graph

562 theory). Here a peer is another process model, which may or may not have one or more child 563 processes. 


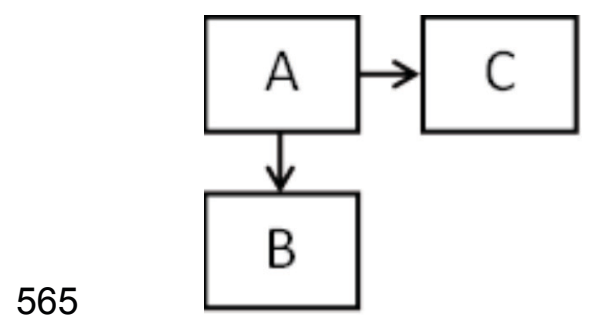

566 Figure 3. Example process model tree with master process model A linked to child B and 567 peer C. Process model B follows A's time stepping (either lock step or through sub-steps) while $568 \quad A$ and $C$ are synchronized at specified points in time. 
570

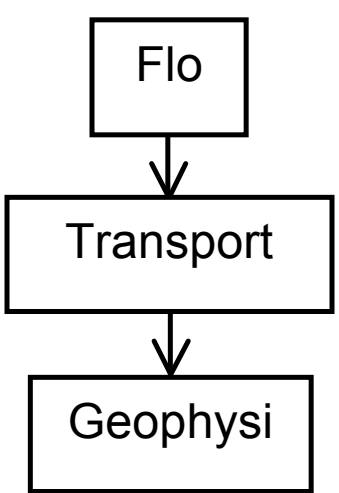

571 Figure 4. The process model tree for PFLOTRAN-E4D coupling where single-phase

572 variably saturated flow is the master process model with solute transport as its child and

573 geophysics is the child of transport. In most cases, all three process models proceed lock step

574 with each other (flow $\rightarrow$ transport $\rightarrow$ geophysics).

575

576 


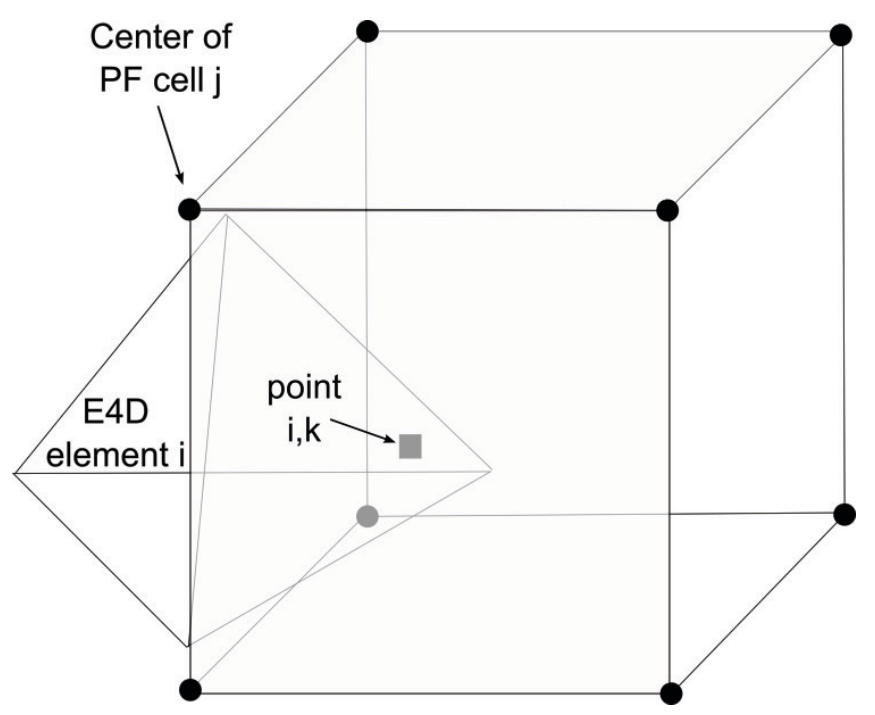




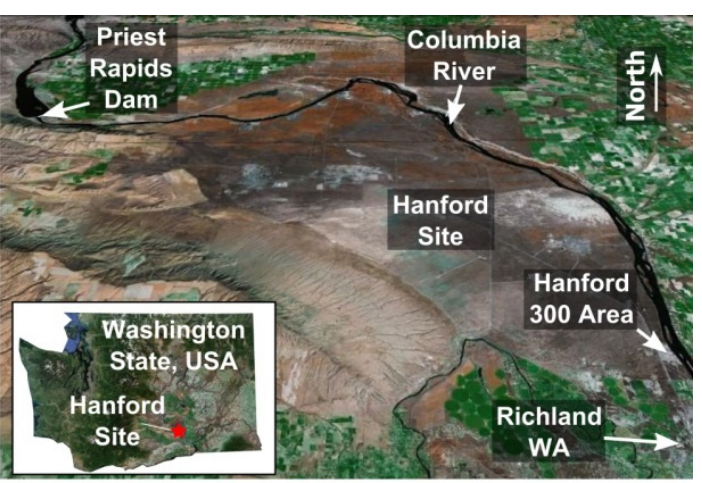

580

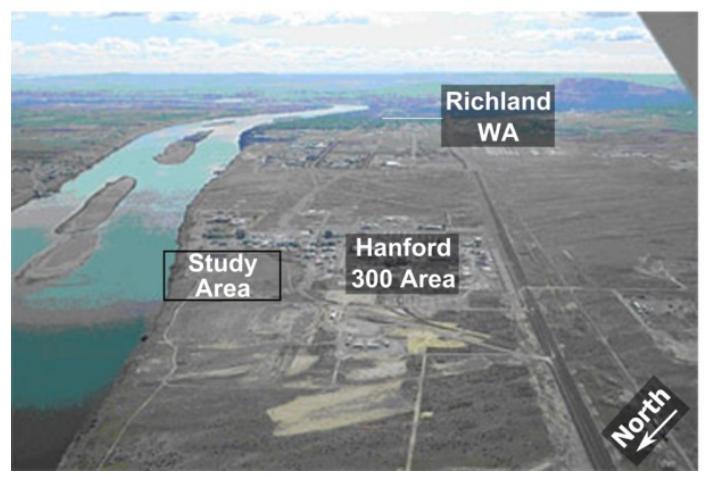

581 Figure 6. Location of simulated study area along the Columbia River at the Hanford 300

582 Area in south-central WA, USA.

583 

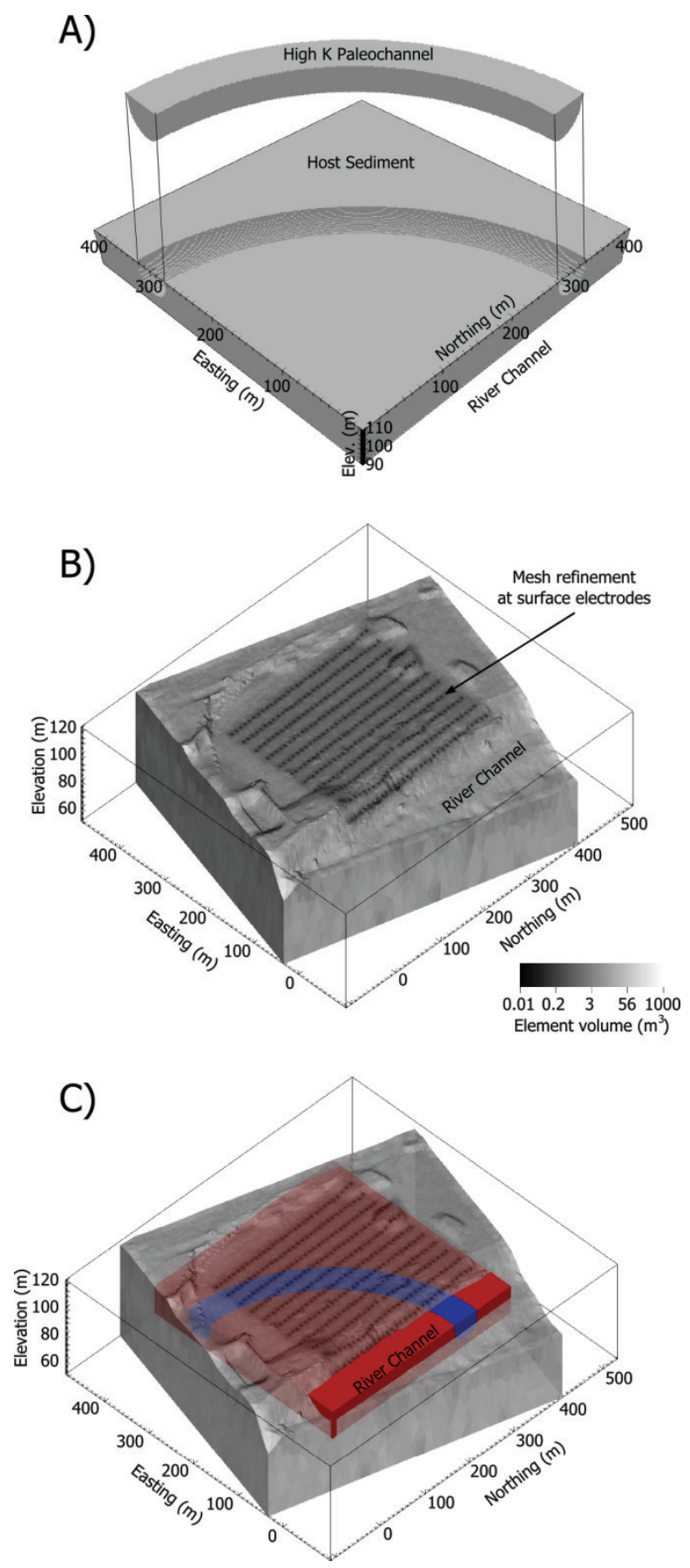

Figure 7. A) PFLOTRAN computational mesh showing a high permeability paleochannel 586 embedded in a lower permeability host sediment. B) Interior E4D mesh showing surface 587 topography and mesh refinement at electrode locations. C) PFLOTRAN mesh (blue channel 
588 and red host material) superimposed on a transparent view of the E4D mesh. 


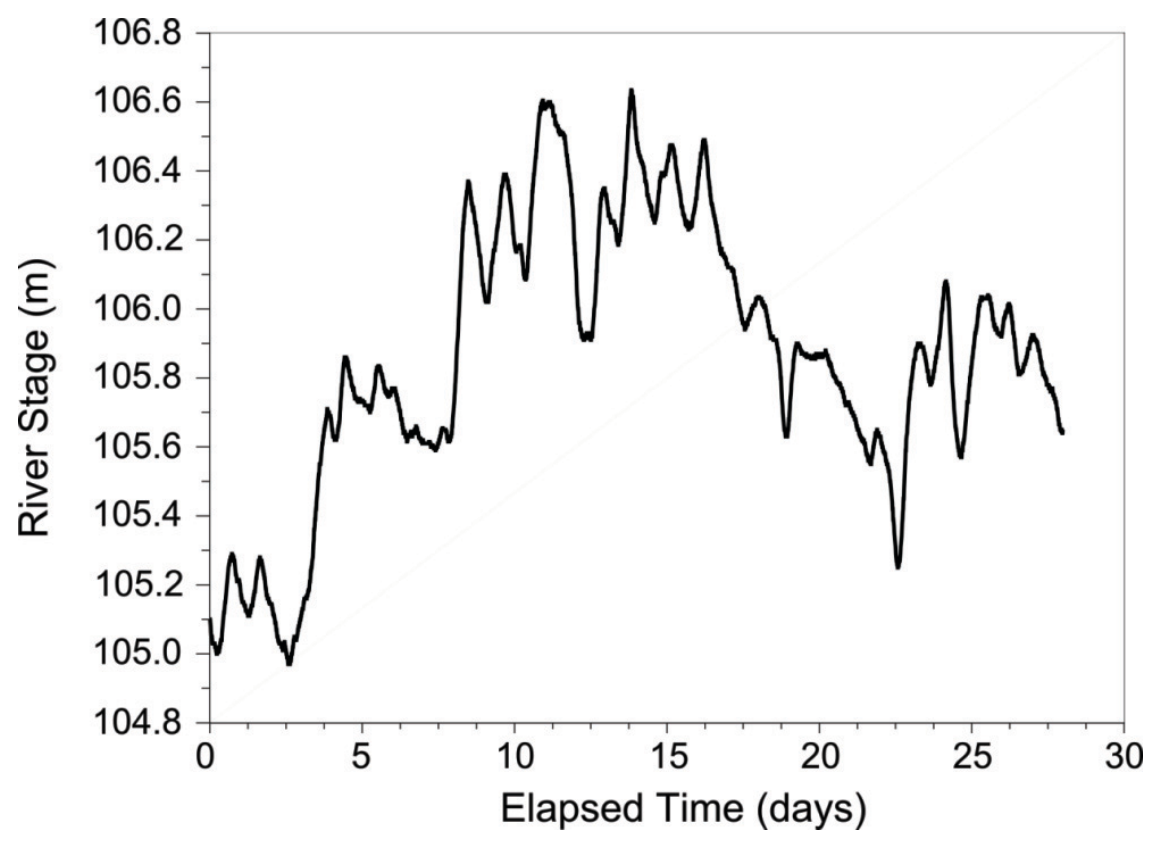

$591 \quad$ Figure 8. River stage during simulation period (April 1 - April 28, 2013).

592 
Time $=5$ days

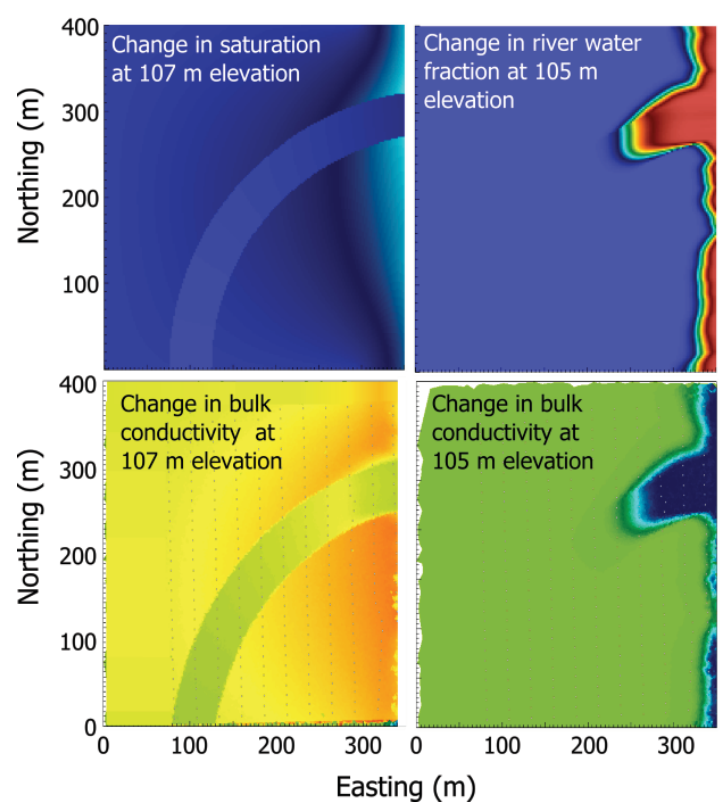

Time $=15$ days
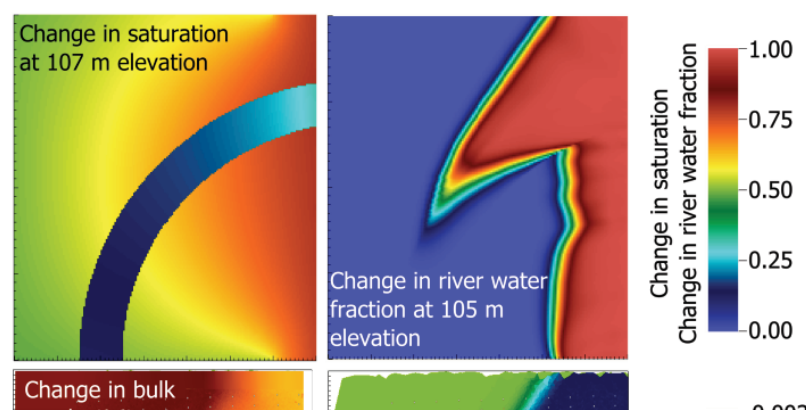

conductivity at

107 m elevation
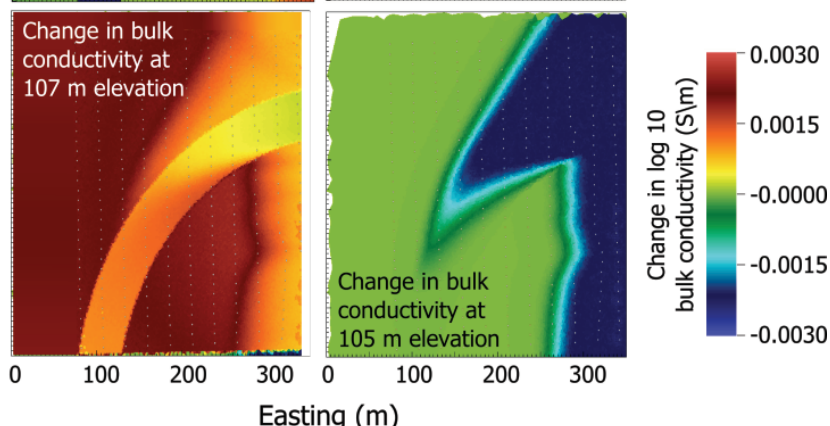

593

Figure 9. (Top Row) Simulated change in saturation and river water fraction from baseline

595 conditions on the PFLOTRAN mesh at 5 and 15 days, and at $105 \mathrm{~m}$ and $107 \mathrm{~m}$ elevation.

596 (Bottom Row) Simulated change in bulk electrical conductivity from baseline conditions at 5 and

59715 days, and at $105 \mathrm{~m}$ and $107 \mathrm{~m}$ elevation.

598

599 


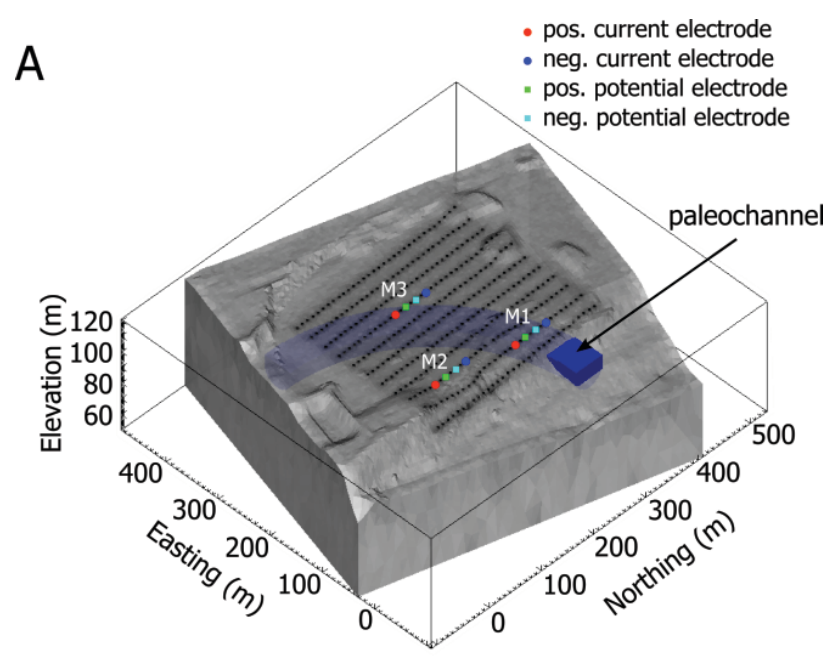

B
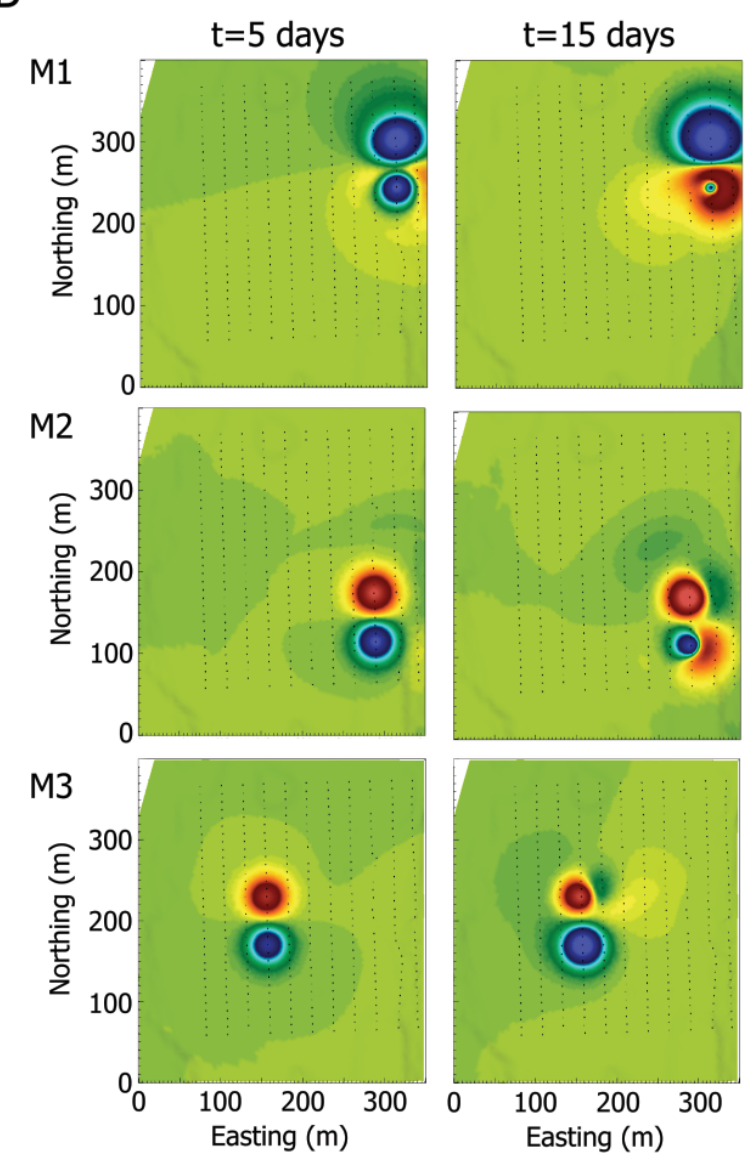

Change in Potential (V/A)

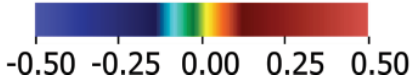


601 Figure 10. A) Locations of the three (M1-M3) 4-electrode Wenner measurements

602 demonstrated in (B) and in figure 8. B) Change in surface potential per ampere of current 603 injected for measurements M1-M3, at 5 and 15 days.

604 
604

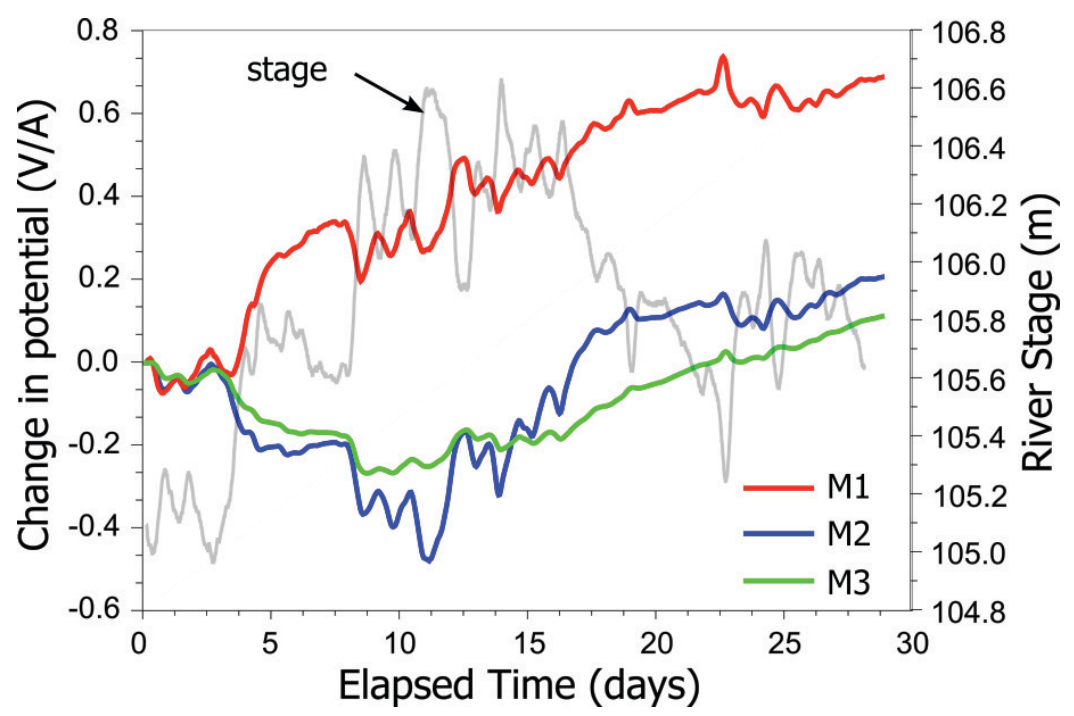

606 Figure 11. Transfer resistance time series for measurements M1-M3 (see figure 7)

607 superimposed on the river stage elevation.

608

609 